\title{
Controling the number of focal elements
}

\author{
Some combinatorial considerations
}

\author{
Christophe Osswald
}

\begin{abstract}
A basic belief assignment can have up to $2^{n}$ focal elements, and combining them with a simple conjunctive operator will need $\mathcal{O}\left(2^{2 n}\right)$ operations. This article proposes some techniques to limit the size of the focal sets of the bbas to be combined while preserving a large part of the information they carry.

The first section revisits some well-known definitions with an algorithmic point of vue. The second section proposes a matrix way of building the least committed isopignistic, and extends it to some other bodies of evidence. The third section adapts the $k$-means algorithm for an unsupervized clustering of the focal elements of a given bba.
\end{abstract}

Key words: Basic belief assignments, Combinatorial complexity, Focal elements, $k$-means, Pignistic probability, Body of evidence, Least commitment

\section{General considerations on basic belief assignments}

Let the finite set $X=\left\{x_{1}, \ldots, x_{n}\right\}$ be our frame of discernment. The size of $X$ will be noted $n=|X|$. The set of all the subsets of $X$ will be noted $2^{X}$.

Definition 1. [Shafer(1976)] The application $m$ from $2^{X}$ to $[0,1]$ is a basic belief assignment (bba) if :

$$
\sum_{A \subseteq X} m(A)=1
$$

The constraint of closed world is modeled by $m(\emptyset)=0$. If $m(\emptyset)$ is greater than 0 , we either have an open world or a conflict within the information.

Christophe OsSWaLD

ENSTA Bretagne, Lab-STICC UMR 3192 e-mail: Christophe.Osswald@ensta-bretagne.fr 
Definition 2. Let $m$ be a bba on $X . A \subseteq X$ is a focal element of $m$ if $m(A)>0$. The focal set of $m$ is composed of all its focal elements :

$$
F(m)=\{A \subseteq X \mid m(A)>0\}
$$

The size of $m$ is noted $|m|=\operatorname{Card}(F(m))$.

Of course, $|m| \leqslant 2^{n}$. In most applications, $|m|$ will be very small compared to $2^{n}$ when a bba is constructed from a source's information, but after some steps of combination, this limit can be reached.

Definition 3. Let $m$ be a bba on $X$. The most usual bodies of evidence are :

- The belief:

$$
\operatorname{bel}(A)=\sum_{\substack{B \subseteq A, B \neq \emptyset}} m(B)=\sum_{\substack{B \subseteq A, B \neq \emptyset, B \in F(m)}} m(B)
$$

- The plausibility:

$$
\operatorname{pl}(A)=\sum_{B \cap A \neq \emptyset} m(B)=\sum_{\substack{B \cap A \neq \emptyset, B \in F(m)}} m(B)
$$

- The commonality:

$$
\mathrm{q}(A)=\sum_{B \supseteq A} m(B)=\sum_{\substack{B \supseteq A \\ B \in F(m)}} m(B)
$$

- The pignisitic probability, which is additive (knowing $\operatorname{betP}(\{x\})$ for all $x \in X$ is sufficient):

$\operatorname{betP}(A)=\frac{1}{1-m(\emptyset)} \sum_{B \subseteq X} \frac{|A \cap B|}{|B|} m(B)=\frac{1}{1-m(\emptyset)} \sum_{B \in F(m)} \frac{|A \cap B|}{|B|} m(B)$

When the context is not obvious, the bba used to define the body of evidence will be placed as an index $: \operatorname{betP}_{m}(A)$ instead of $\operatorname{betP}(m)$.

In the definition 3, the first expression concerns all the subsets of $X$, and the second expression concerns only the focal elements. Therefore, if $f$ is either of the bodies of evidence, and $A$ a subset of $X$, a natural implementation of the equation brings an algorithm which calculates $f(A)$ in $\mathcal{O}\left(2^{n}\right)$ operations with the first expression. As the second expression only browses the focal set of $m$, its complexity is $\mathcal{O}(|m|)$, for the same result.

The most popular combination operator is the non-normalized conjunctive rule, also known as Smet's rule. It is a quite simple operator to implement; it is associative, and therefore allows to combine many sources. 
Definition 4. Let $m_{1}$ and $m_{2}$ be two bbas on $X$. The conjunctive combination of $m_{1}$ and $m_{2}$ is a bba on $X, m_{1} \oplus m_{2}$, defined by :

$$
\left(m_{1} \oplus m_{2}\right)(A)=\sum_{\substack{B \subseteq X, C \subseteq X, B \cap C=A}} m_{1}(B) m_{2}(C)=\sum_{\substack{B \in F\left(m_{1}\right), C \in F\left(m_{2}\right), B \cap C=A}} m_{1}(B) m_{2}(C)
$$

The cost for calculating $B \cap C$ is $\mathcal{O}(n)$. The first expression brings an algorithm in $\mathcal{O}\left(n 2^{2 n}\right)$ operations for calculating $\left(m_{1} \oplus m_{2}\right)(A)$, and $\mathcal{O}\left(n 2^{3 n}\right)$ for determining $m_{1} \oplus m_{2}$. The second expression brings an algorithm in $\mathcal{O}\left(n\left|m_{1}\right|\left|m_{2}\right|\right)$ operations for calculating $\left(m_{1} \oplus m_{2}\right)(A)=\left(m_{1} \oplus m_{2}\right)(A)$, and $\mathcal{O}\left(n 2^{n}\left|m_{1}\right|\left|m_{2}\right|\right)$ for determining $m_{1} \oplus m_{2}$.

Smets $[\operatorname{Smets}(2002)]$ proposed a nice implementation in $\mathcal{O}\left(n 2^{n}\right)$ operations for transformations between bba and commonality. The conjunctive combination of the commonality functions is a simple multiplication, which is linear, but on vectors having a size of $2^{n}$.

The expression (7), nor the commonality, can prevent us from making operations on non-focal elements of $m_{1} \oplus m_{2}$. Let the bba be implemented by an adaptive structure that contains information only for its focal elements. A hashtable is a convenient way for it. The algorithm 1 uses only $\mathcal{O}\left(n\left|m_{1}\right|\left|m_{2}\right|\right)$ to build $m_{1} \oplus m_{2}$.

The size of $m_{\cap}$ is at most $\left|m_{1}\right|\left|m_{2}\right|$. The algorithm coming from (7) needs to be executed for all the subsets of $X$, but the algorithm 1 only works on the focal elements of $m_{\cap}$, and does not compute useless intersections [Smets(1994)]. Using a hashtable for the focal elements, with a hashcode calculation in $\mathcal{O}(n)$ operations, the conjunctive combination takes $\mathcal{O}\left(n\left|m_{1}\right|\left|m_{2}\right|\right)$ operations.

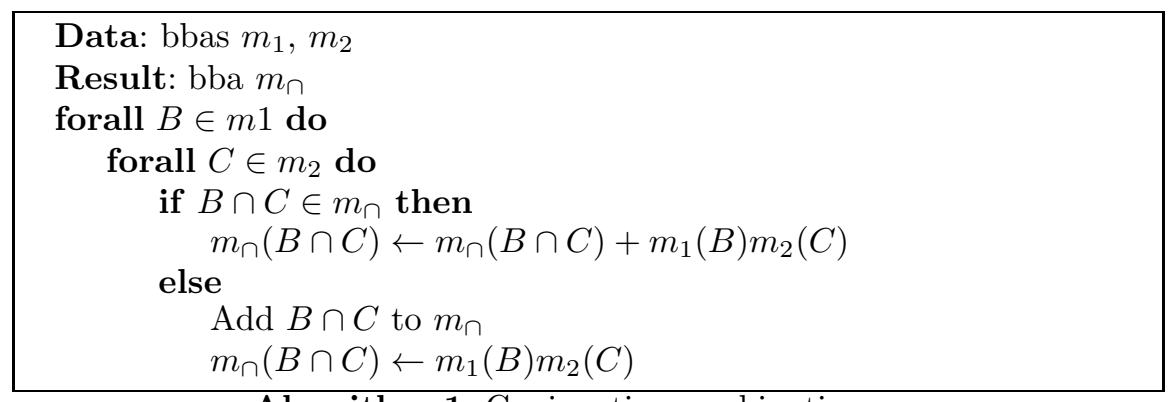

Algorithm 1: Conjunctive combination

However, the very nature of the combination operator brings a combinatorial explosion of the focal set. Let $m_{i}$ be the bba defined by $m_{i}(X)=\frac{1}{2}$ and $m_{i}\left(X \backslash\left\{x_{i}\right\}\right)=\frac{1}{2}:\left|m_{i}\right|=2$. Let $m_{\cap}$ be the conjunctive combination of all those bbas : $m_{\cap}=m_{1} \oplus \ldots \oplus m_{n}$. For any $A \subseteq X, m_{\cap}(A)=\frac{1}{2^{n}}$. Therefore, $F\left(m_{\cap}\right)=2^{X}$ and $\left|m_{\cap}\right|=2^{n}$.

The objective of the following sections will be to guarantee that the size of a bba cannot be too large, and to respect its nature as much as possible. 


\section{Linear algebra for bbas}

The definition 3 builds the bodies of evidence bel, pl, betP and $\mathrm{q}$ as linear transformations of $m$. Considering a bba $m$ on $X$ and an integer $K$, our objective will be to build an bba $m^{\prime}$ on $X$ such that $\left|m^{\prime}\right| \leqslant K$ and $f_{m^{\prime}}(A)=$ $f_{m}(A)$ for some bodies of evidence $f$ and some subsets $A$ of $X$.

Within this section, we forbid $\emptyset$ to be a focal element of $m$, and we do not allow it to become a focal element of $m^{\prime}$. As convenient consequences, we have $\operatorname{bel}(A) \leqslant \operatorname{betP}(A) \leqslant \operatorname{pl}(A), \operatorname{bel}(X)=1$, and $\operatorname{pl}(X)=1$.

A popular and efficient way to build a bba from a probability or another source of uncertain information is to build a least committed bba having the same pignistic probability than the source [Smets(1990)].

Definition 5. Let $m$ be a bba on $X$. A bba $m^{\prime}$ is an isopignisitic of $m$ if

$$
\forall x \in X, \operatorname{betP}_{m}(x)=\operatorname{betP}_{m^{\prime}}(x)
$$

The bba $m^{\prime}$ is the least committed isopignistic of $m$ if for any isopignistic $m^{\prime \prime}$ of $m$ and for any $A \subseteq X, \mathrm{pl}_{m^{\prime}}(A) \geqslant \mathrm{pl}_{m^{\prime \prime}}(A)$.

The algorithm 2 builds the least committed isopignistic in $\mathcal{O}\left(n^{2}+n|m|\right)$ operations. It contains at most $n$ focal elements.

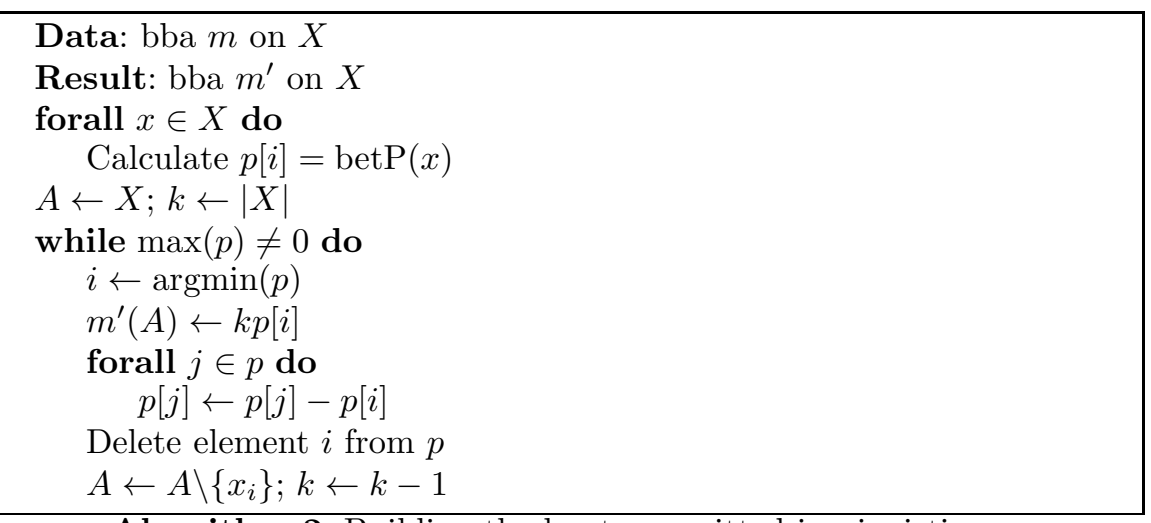

Algorithm 2: Building the least committed isopignistic

If we calculate $\operatorname{betP}(x)$ for all $x \in X$, and order the elements of $X$ such that $p_{i}=\operatorname{betP}\left(x_{i}\right) \geqslant \operatorname{betP}\left(x_{i+1}\right)=p_{i+1}$, the focal elements of the least committed isopignistic are a subset of the $A_{i}=\left\{x_{1}, \ldots, x_{i}\right\}$.

We have

$$
p_{i}=\operatorname{betP}\left(x_{i}\right)=\sum_{k=i}^{n} \frac{1}{k} m^{\prime}\left(A_{k}\right)
$$

Let $p$ be the vector of the $p_{i}$ and $y$ be the vector of the $m^{\prime}\left(A_{i}\right)$. We have $p=\operatorname{Bet} y$ with Bet a $n \times n$ matrix, triangular and inversible. Therefore 
$y=\operatorname{Bet}^{-1} p$, with

$$
\operatorname{Bet}=\left(\begin{array}{cccccc}
1 & \frac{1}{2} & \frac{1}{3} & \cdots & \frac{1}{n-1} & \frac{1}{n} \\
0 & \frac{1}{2} & \frac{1}{3} & \cdots & \frac{1}{n-1} & \frac{1}{n} \\
\vdots & 0 & \frac{1}{3} & \cdots & \frac{1}{n-1} & \frac{1}{n} \\
\vdots & & \ddots & \ddots & & \vdots \\
\vdots & & & \ddots & \frac{1}{n-1} & \vdots \\
0 & \cdots & \cdots & \cdots & 0 & \frac{1}{n}
\end{array}\right), \operatorname{Bet}^{-1}=\left(\begin{array}{cccccc}
1 & -1 & 0 & \cdots & \cdots & 0 \\
0 & 2 & -2 & 0 & & \vdots \\
0 & 0 & 3 & -3 & \ddots & \vdots \\
\vdots & & \ddots & \ddots & \ddots & 0 \\
\vdots & & & \ddots & (n-1) & -(n-1) \\
0 & \cdots & \cdots & \cdots & 0 & n
\end{array}\right)
$$

As Bet ${ }^{-1}$ is a triangular band matrix, we can compute all the $m^{\prime}\left(A_{i}\right)$ from $p_{i}$ in $\mathcal{O}(n)$ operations.

With $\mathcal{O}(n|m|)$ operations for computing betP, $\mathcal{O}(n \ln n)$ operations for sorting $X, \mathcal{O}(n)$ operations for building the sets $A_{i}$ (with an adapted data structure) and $\mathcal{O}(n)$ operations for solving the linear system, building the least committed isopignistic costs $\mathcal{O}(n(\ln n+|m|))$ operations. Usually, $|m| \gg \ln n$, and the cost of the least committed isopignistic is not greater than the cost of computing betP $(x)$ for the elements of $X$.

The interval $[\operatorname{bel}(A), \operatorname{pl}(A)]$, containing betP $(A)$, can be interpretated as an uncertainty on $A[\mathrm{Janez}$ and Appriou(1996)]. For singletons, bel is trivial: $\operatorname{bel}(x)=m(x)$. For sets of size $n-1, \mathrm{pl}$ is trivial: $\operatorname{pl}(X \backslash\{x\})=1-m(\{x\})$. Considering the non-trivial bodies of evidence on the sets of interest $\left\{x_{1}\right\}$, $\ldots,\left\{x_{n}\right\}, B_{1}=X \backslash\left\{x_{1}\right\}, \ldots, B_{n}=X \backslash\left\{x_{n}\right\}$, we search a bba $m^{\prime}$ with those focal elements, forming a vector

$$
\mathbf{y}=\left(m^{\prime}\left(\left\{x_{1}\right\}\right), \ldots, m^{\prime}\left(\left\{x_{n}\right\}\right), m^{\prime}\left(B_{1}\right), \ldots, m^{\prime}\left(B_{n}\right)\right)^{T}
$$

which verifies:

$$
\begin{aligned}
& \forall i \in\lfloor 1, n\rfloor, \operatorname{pl}_{m^{\prime}}\left(\left\{x_{i}\right\}\right)=\operatorname{pl}_{m}\left(\left\{x_{i}\right\}\right) \\
& \forall i \in\lfloor 1, n\rfloor, \operatorname{bel}_{m^{\prime}}\left(B_{i}\right)=\operatorname{bel}_{m}\left(B_{i}\right)
\end{aligned}
$$

We have:

$$
\begin{aligned}
& \operatorname{pl}_{m^{\prime}}\left(\left\{x_{i}\right\}\right)=m^{\prime}\left(\left\{x_{i}\right\}\right)+\sum_{j \neq i} m^{\prime}\left(B_{j}\right) \\
& \operatorname{bel}_{m^{\prime}}\left(B_{i}\right)=\sum_{j \neq i} m^{\prime}\left(\left\{x_{j}\right\}\right)+m^{\prime}\left(B_{i}\right)
\end{aligned}
$$

As $\forall i, \mathrm{pl}_{m^{\prime}}\left(\left\{x_{i}\right\}\right)+\operatorname{bel}_{m^{\prime}}\left(B_{i}\right)=\sum_{i} m^{\prime}\left(\left\{x_{i}\right\}\right)+\sum_{i} m^{\prime}\left(B_{j}\right)$, there are only $n+1$ independent equations among the $2 n$ listed above: we cannot guarantee to kep at the same time $\mathrm{pl}_{m}\left(\left\{x_{i}\right\}\right)$ and $\operatorname{bel}_{m}\left(B_{i}\right)$ on those $2 n$ focal elements.

As $\mathrm{q}\left(B_{i}\right)=m\left(B_{i}\right)+m(X)$ and $\mathrm{q}\left(\left\{x_{i}\right\}\right)=\operatorname{pl}\left(\left\{x_{i}\right\}\right)$, introducing commonality does not bring any new independent equation. 


\subsection{Mixing Bet with other bodies of evidence}

Here we search a bba with $2 n$ focal elements which is an isopignistic of $m$ and respects an other body of evidence on some focal elements. In the following examples, we allow the $A_{i}$ obtained in section 2 to be focal elements, and we complete them with $\left(\left\{x_{i}\right\}\right)_{i \in\lfloor 1, n\rfloor}$ or the $\left(B_{i}\right)_{i \in\lfloor 1, n\rfloor}$.

With plausibility, we should use the focal elements $\left(\left\{x_{i}\right\}\right)_{i \in\lfloor 1, n\rfloor}$. We build a vector

$$
\mathbf{y}=\left(m^{\prime}\left(\left\{x_{1}\right\}\right), \ldots, m^{\prime}\left(\left\{x_{n}\right\}\right), m^{\prime}\left(A_{1}\right), \ldots, m^{\prime}\left(A_{n}\right)\right)^{T}
$$

The constraints are:

$$
\begin{gathered}
\operatorname{betP}\left(x_{i}\right)=m^{\prime}\left(\left\{x_{i}\right\}\right)+\sum_{k=i}^{n} \frac{1}{k} m^{\prime}\left(A_{k}\right) \\
\operatorname{pl}\left(\left\{x_{i}\right\}\right)=m^{\prime}\left(\left\{x_{i}\right\}\right)+\sum_{k=i}^{n} m^{\prime}\left(A_{k}\right)
\end{gathered}
$$

As $A_{1}=\left\{x_{1}\right\}$, we cannot have $m^{\prime}\left(A_{1}\right) \neq m^{\prime}\left(\left\{x_{1}\right\}\right)$; we have only $2 n-1$ focal elements. We drop the term $m^{\prime}\left(\left\{x_{1}\right\}\right)$ in $y$, and the constraint on $\operatorname{pl}\left(\left\{x_{i}\right\}\right)$ to obtain a matrix $P$ such that

$$
P \mathbf{y}=\left(\mathrm{pl}_{m}\left(\left\{x_{2}\right\}\right), \ldots, \operatorname{pl}_{m}\left(\left\{x_{2}\right\}\right), \operatorname{betP}\left(x_{1}\right), \ldots, \operatorname{betP}\left(x_{n}\right)\right)^{T}
$$

The matrix $P_{4}$ and more generally $P_{n}$ are:

$$
P_{4}=\left(\begin{array}{ccccccc}
0 & 0 & 0 & 1 & \frac{1}{2} & \frac{1}{3} & \frac{1}{4} \\
1 & 0 & 0 & 0 & \frac{1}{2} & \frac{1}{3} & \frac{1}{4} \\
0 & 1 & 0 & 0 & 0 & \frac{1}{3} & \frac{1}{4} \\
0 & 0 & 1 & 0 & 0 & 0 & \frac{1}{4} \\
1 & 0 & 0 & 0 & 1 & 1 & 1 \\
0 & 1 & 0 & 0 & 0 & 1 & 1 \\
0 & 0 & 1 & 0 & 0 & 0 & 1
\end{array}\right), \quad P_{n}=\left(\begin{array}{cc|c|c}
\frac{0 \cdots}{I_{n-1}} & \multirow{2}{*}{\operatorname{Bet}_{n}} \\
& & \\
& I_{n-1} & \vdots & U_{n-1} \\
& & 0 &
\end{array}\right)
$$

where $\operatorname{Bet}_{n}$ is matrix obtained in the section 2 and $U_{n-1}$ the upper triangular $(n-1) \times(n-1)$ matrix full of 1 .

The matrix $P_{n}$ is inversible, and we can solve this system in $\mathcal{O}\left(n^{3}\right)$ operations. Overall, we can reduce the focal set of $m$ to $2 n-1$ focal elements in $\mathcal{O}\left(n\left(n^{2}+|m|\right)\right)$ operations, respecting betP and $\mathrm{pl}$ on the singletons.

With commonality, we obtain the same results : $\mathrm{q}\left(\left\{x_{i}\right\}\right)=\operatorname{pl}\left(\left\{x_{i}\right\}\right)$.

With belief, we should use $\left(B_{i}\right)_{i \in\lfloor 1, n\rfloor}$ as focal elements instead of $\left(\left\{x_{i}\right\}\right)$. As bel $\left(B_{i}\right)+\operatorname{pl}\left(\left\{x_{i}\right\}\right)=1$, we obtain another - but similar $-(2 n-1) \times(2 n-1)$ inversible matrix. 


\section{Optimatization by $k$-means}

[Denoeux and Yaghlane(2002)] proposed to reduce a bba by adapting the single linkage hierarchical clustering algorithm to coarsen its focal set. Another interesting family of unsupervized clustering algorithm are the $k$-means techniques, born from the ISODATA method of [Ball and Hall(1965)]. One can adapt this method to find a subset $\mathcal{K}$ of $2^{X}$ limited in size: $|\mathcal{K}| \leqslant k$.

Usual $k$-means does not guarantee an optimal choice of centers: finding them is equivalent to the MINIMUM- $k$ CENTER, which is a NP-Complete problem [Garey and Johnson(1979)]. The convergence of the $k$-means algorithm is guaranteed, but only to a local minimum of the intra-cluster variance.

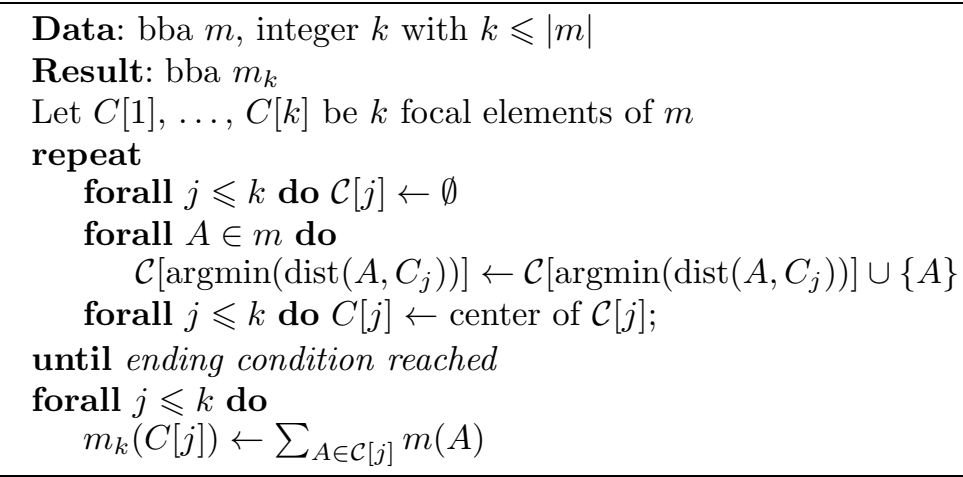

Algorithm 3: $k$-means, in a general way that applies to focal elements.

[1] It is natural to initialize the algorithm with the $k$ focal elements with the greatest masses. But, as the algorithm converges - if it converges - to a local minimum, it should be a good idea to execute various instances, with random starting sets.

[2] The focal element $A$ is affected to the center $C[j]$ such that

$$
\operatorname{dist}(A, C[j])=|(A \cap \overline{C[j]}) \cup(\bar{A} \cap C[j])|
$$

is minimal. It corresponds to a natural $L_{1}$ distance based on an exclusive OR. In case of equal distances to different centers, it is possible to:

- choose a random one

- use a lexicographical order

(the algorithm is no longer deterministic)

- try to build balanced clusters (the (elements are no longer equivalent)

[3] The usual $k$-means technique uses the geometrical barycenter of the focal sets of $\mathcal{C}[j]$ seen as points of $[0,1]^{n}: C[j] \leftarrow \sum_{A \in \mathcal{C}[j]} m(A) A$.

It would build fuzzy focal elements, which is not the way the definition 2 accepts them. Therefore, we put $x$ in the new $C[j]$ if and only if :

$$
\sum_{A \in \mathcal{C}[j], x \in A} m(A)>\sum_{A \in \mathcal{C}[j], x \notin A} m(A)
$$


[4] As we "move" the centers of the classes to the nearest sharp subset of $X$, the total intra-cluster variance is not necessarily decreasing. Therefore, the ending condition must include a maximum steps number, and/or test the cycles it should encounter.

A step of the algorithm 3 costs $\mathcal{O}(k n|m|)$ operations. A reasonable number of steps before ending the loop is $k$, and we obtain an algorithm in $\mathcal{O}\left(k^{2} n|m|\right)$ operations. If we want to compare this approach with the ones of the section 2.1 , we should use $k=2 n-1$, and get an algorithm in $\mathcal{O}\left(n^{3}|m|\right)$ operations.

\section{Conclusion}

In a general way, dealing with basic belief assignments on large frames of discernment need a proper encoding of the focal sets. We propose to use hashtables for this purpose, but this not the only way. We propose two categories of methods for restricting any bba to a bba modest in focal set size.

We extend the principle of isopignistic to other bodies of evidence to build a bba with only $2 n-1$ focal elements, respecting both the pignistic probability and another body of evidence of the original bba. We first determine the value of the bodies of evidence on some simple elements, and then determine the restricted focal set. A linear equation gives the restricted bba.

Trying to restrict the focal set to a number of respresentative elements leads to a NP-Complete problem. We adapt the $k$-mean algorithm to build a heuristical solution. It is more expensive, but it does not need to define $a$ priori a focal set, and can adapt to more situations.

\section{References}

[Ball and Hall(1965)] Ball GH, Hall DJ (1965) Isodata, a novel methed of data analysis and pattern classification. Tech. rep., Stanford Research Institute

[Denoeux and Yaghlane(2002)] Denoeux T, Yaghlane AB (2002) Approximating the combination of belief functions using the fast moebius transform in a coarsened frame. International Journal of Approximate Reasoning 31(1-2):77-101

[Garey and Johnson(1979)] Garey MR, Johnson DS (1979) Computers and intractability - a guide to the theory of NP-Completeness. Freeman

[Janez and Appriou(1996)] Janez F, Appriou A (1996) Théorie de l'Evidence et cadres de discernement non exhaustifs. Traitement du Signal 13(3):237-250

[Shafer(1976)] Shafer G (1976) A mathematical theory of evidence. Princeton University Press

[Smets(1990)] Smets P (1990) Constructing the pignistic probability function in a context of uncertainty. Uncertainty in Artificial Intelligence 5:29-39

[Smets(1994)] Smets P (1994) The transferable belief model. Artificial Intelligent 66:191234

[Smets(2002)] Smets P (2002) Matrix Calculs for Belief Functions. International Journal of Approximate Reasoning 31:1-30 